\title{
The Independent, Joint, and Additive Associations of Physical Activity and Self-Compassion on Depression Symptoms Among Chinese College Students
}

\author{
Liuyue Huang $\mathbb{( D}^{1, *}$ \\ Kaixin Liang $\mathbb{D}^{1, *}$ \\ Si-Tong Chen ${ }^{2}$ \\ Yizhen Ren $^{3}$ \\ Yi Zhu ${ }^{4}$ \\ Xinli Chi ${ }^{1}$
}

'School of Psychology, Shenzhen University, Shenzhen, 5I806I, People's Republic of China; ${ }^{2}$ Institute for Health and Sport, Victoria University, Melbourne, 800I, Australia; ${ }^{3}$ Beijing Key Laboratory of Applied Experimental Psychology, Faculty of Psychology, Beijing Normal University, Beijing, I00875, People's Republic of China; ${ }^{4}$ School of Early-Childhood Education, Nanjing Xiaozhuang University, Nanjing, 2 I I I7I, People's Republic of China

*These authors contributed equally to this work
Correspondence: Yi Zhu; Xinli Chi Email16187654@qq.com; xinlichi@szu. edu.cn
Background: Physical activity and self-compassion are closely related to individuals' mental health (eg, depression). However, most studies only examined their independent roles in mental health without considering the potential interaction between the two variables. The present study aimed to investigate the independent and joint associations between physical activity (PA) and self-compassion (SC) on depression symptoms and examine the additive interaction between PA and SC.

Methods: A cross-sectional design was utilized in the present descriptive study. By recruiting participants via social media platforms, 1846 Chinese college students completed International Physical Activity Questionnaire Short Form, the Self-Compassion Scale-Short Form, the 9-item Patient Health Questionnaire, and items regarding socio-demographic information. Independent and joint roles of low-level PA (LPA) and low-level SC (LSC) on depression symptoms were examined by logistic regression models. Additive interaction between LPA and LSC on depression symptoms was examined by the following indices: relative excess risk due to interaction (RERI), attributable proportion (AP), and synergy index (S). Socio-demographic variables were included in the models as covariates.

Results: Both LPA $(\mathrm{OR}=1.47[1.16,1.86])$ and $\operatorname{LSC}(\mathrm{OR}=5.74[3.89,8.45])$ were independently associated with higher probability of depression symptoms. Combining LPA and LSC led to an additive interaction and greatly increased the odds of depression symptoms $(\mathrm{OR}=9.62[5.38,17.22] ; \mathrm{RERI}=2.47[0.19,4.75], \mathrm{AP}=0.25[0.05,0.44]$, $\mathrm{S}=1.38[1.02,1.87])$.

Conclusion: Both LPA and LSC were associated with an increased risk of depression symptoms. Moreover, combining LPA and LSC may develop an additive risk for depression symptoms. Future research and clinical intervention could integrate PA and SC to find a better way to resist depression symptoms.

Keywords: physical activity, self-compassion, depression, interaction, college students

\section{Introduction}

Depression symptoms among youths capture considerable attention from researchers and practitioners. Numerous studies have indicated the high prevalence of depression symptoms among youths. For instance, a meta-analysis covering 45 studies including 50,825 participants revealed that the prevalence of depression symptoms among Chinese college students was about $30.39 \%{ }^{1}$ Depression is a psychosomatic phenomenon showing both physical and psychological symptoms 
(eg, emotional and physical retarded activity), ${ }^{2}$ and it associates with an array of detrimental health outcomes among young adults, including impaired cognitive processes, a variety of somatic diseases, and even frequent suicidal ideation and suicide attempts. ${ }^{3}$ Given the prevalence and adverse influence of depression symptoms, it is essential to reveal factors that could provide more targeted and efficient interventions. The present research focuses on two important factors, namely physical activity (PA) and self-compassion (SC).

The role of lifestyle factors (eg, physical activity [PA]) in the intervention of psychopathology (eg, depression) has been gaining increasing attention among researchers (eg, lifestyle psychiatry framework ${ }^{4,5}$ ). Many studies have established a consistent association between PA and mental health outcomes in young people, including depression symptoms. ${ }^{6,7}$ For instance, research suggests that insufficient PA associates with the risk of depression symptoms. $^{8,9}$ From the perspective of the stressvulnerability model of depression, low-level PA (LPA) may increase the vulnerability towards depression biologically by dysregulating the generation of some hormones $^{10}$ and psychologically by increasing negative emotion. $^{11}$ According to self-determination theory (SDT), ${ }^{12}$ human behaviors are driven by three basic psychological needs, namely, autonomy, competence, and relatedness. Physical activity is conducive to promote selfefficacy, ${ }^{13}$ and further help individuals to meet the essential need of autonomy and competence. In addition, exercise and sports, forms of physical activity, might involve social interaction with others, cultivating the sense of relatedness.

Self-compassion (SC) is another factor that emerges as an effective intervention approach for mental health and well-being. ${ }^{14}$ SC emphasizes the autonomy and competence of "self" to be kind towards oneself in suffering, and it views sufferings as shared human experiences with a sense of relatedness. Thus, $\mathrm{SC}$ is also closely linked to human psychological needs proposed by SDT. SC can intervene with the vicious cognitive-emotional cycle and alleviate depression symptoms. ${ }^{15}$ Research suggests that $\mathrm{SC}$ is a strong predictor of depression, ${ }^{14}$ suggesting that it is effective for mental health and stress management. For instance, low-level self-compassion (LSC) has been shown to be linked to multiple kinds of mental illness indicators $^{15,16}$ and may predispose people to be vulnerable to depression symptoms. ${ }^{17,18}$
The above information suggest that SC and PA are tightly associated with people's mental health. Specifically, the present study proposed that LPA (Hypothesis 1a) and LSC (Hypothesis 1b) were positively associated with depression symptoms among Chinese college students, respectively.

In the field of health care, instead of solely relying on external resources, researchers and practitioners focus more on the importance of self-care and the value of individuals' sense of agency. SC and PA are constructive ways for self-help. While most studies focus on the independent role of PA and SC in the intervention of depression symptoms, their combined effect is rarely studied. Notably, as proposed by the cumulative risk model, instead of examining a single risk factor, the accumulation of risk factors may better explain an individual's developmental outcomes. ${ }^{19,20}$ Moreover, there is a reciprocal relationship between PA and SC, suggesting that combining PA and SC may produce an additive effect on mental health. On the one side, individuals with high-level self-compassion (HSC) are likely to have a better sense of autonomy for health. ${ }^{21}$ Therefore, individuals with HSC may have more intrinsic motivation (eg, for their physical and mental health) to engage in PA and maintain PA habits. ${ }^{26,27} \mathrm{SC}$ is also associated with more adaptive self-regulation, conducive to rational goal-setting of PA. This may facilitate PA by increasing the individual's sense of competence or self-efficacy with appropriate exercise goals. ${ }^{22,23}$ On the other side, participating in PA is beneficial for regulating emotion and reducing the risk of psychopathology (eg, depression). ${ }^{24,25}$ Individuals may experience less rumination while exercising, which further promotes mindfulness and increase self-compassion. ${ }^{26}$ Indeed, a recent metaanalysis covering 25 studies have concluded that higher levels of PA were associated with higher levels of SC. ${ }^{26}$ Considering the known separate role of LPA and LSC in depression symptoms, along with the mutually reinforcing relationship between $\mathrm{PA}$ and $\mathrm{SC}$, it is thus reasonable to speculate that concurrent exposures to LPA and LSC would generate an additional risk in depression symptoms. Specifically, we proposed that a combination of LPA and LSC was associated with a higher risk of depression symptoms (Hypothesis 2), and there was an additive interaction between LPA and LSC (Hypothesis 3).

Therefore, the purpose of this study was threefold: (1) to examine the independent roles of LPA and LSC on depression symptoms; (2) to investigate the joint roles of LPA and LSC on depression symptoms; (3) and to test 
whether LPA and LSC had an additive interaction in increasing the odds of depression symptoms. Although the independent associations between PA and SC with depression symptoms have been well established, existing studies rarely considered the potential interaction between PA and SC. Besides, studies on the roles of SC in Chinese samples are still limited. Findings from this study would add evidence to the knowledge base of the role of modifiable self-determined factors (SC and PA) on depression symptoms in Chinese samples, and thus may provide theoretical and practical implications for future intervention.

\section{Method}

\section{Participants and Procedure}

The cross-sectional study was conducted via an online survey in August (21-31st) 2020, when the pandemic was under control in China, and college students were about to back to school for the fall semester. A convenient sampling method was adopted in recruiting college students as our participants via social media platforms (ie, WeChat and QQ). The inclusion criteria for participants were: (a) Chinese college students; (b) willing to participate in the study, (c) able to communicate in Chinese, and (d) no major mental health problems at present. Students who had completed all questionnaires (approximately 15 minutes) were given ten RMB (Chinese currency, equivalent to 1.5 USD) via online payment. In total, 1942 participants from 30 provinces and autonomous regions were recruited and 1846 participants (response rate $=95.1 \%$ ) provided valid answers in this study. Participants were asked to provide online consent before filling out the survey. Based on existing studies on depression symptoms during the COVID-19 pandemic, ${ }^{28-31}$ the sample was computed using $G^{*}$ Power 3.1 based on the following assumptions: an expected prevalence $(25.0 \%)$ of symptoms (PHQ-9 scores $\geq 10$ as the cut-off), 1.5 odds ratio (OR) of symptoms among LPA or LSC, margin of error of $5 \%$ and power of $90 \%$, two-tailed examination, and binomial distribution of independent variables. This gives a total sample size of 1435 . The finally recruited sample $(n=1846)$ exceeds this, indicating the sufficiency for statistical analysis. The confidence level was set at 95\%. The research obtained ethics approval from the local Human Research Ethics Committee (blinded for review). All participants were informed about the purpose of the study, and that it was conducted in accordance with the Declaration of Helsinki.

\section{Instruments}

\section{Socio-Demographic Variables}

Socio-demographic variables were collected, including gender (male/female), age (years), body mass index (BMI, calculated by the formula weight $[\mathrm{kg}] /$ height $^{2}$ $[\mathrm{m}]$ ), family structure (full/divorced/other), number of siblings (none/one or more), number of friends (none/1-2/3$5 / 6$ or more), and residence (rural/urban).

\section{Physical Activity (PA)}

PA was self-reported using the International Physical Activity Questionnaire Short Form (IPAQ-SF). ${ }^{32,33}$ Respondents were asked to report the frequency and duration of participating PA of different intensities (highintensity PA, moderate-intensity PA, and low-intensity PA) in the past week. PA refers to activities that involve the contraction of large skeletal muscles, including exercise, sports, walking, activities done at work or in spare time, etc. Example question: "During the last 7 days, on how many days did you do vigorous physical activities like heavy lifting, digging, aerobics, or fast bicycling?" The Chinese version of the IPAQ has been confirmed adequately reliable and valid for measuring total PA in a Chinese population. ${ }^{33}$ Following the scoring rule of IPAQ-SF, participants were classified as low-level PA (less than 600 metabolic equivalents of task (METs) min/ week) and high-level PA (no less than 600 METs min/ week) according to their amount of PA.

\section{Self-Compassion (SC)}

The Self-compassion Scale-Short Form (SCS-SF) was used to measure the level of SC. ${ }^{34}$ It consisted of 12 items, with each item is rated on a 5-point scale $(1=$ almost never to $5=$ almost always). SCS-SF consisted of six subscales: three compassionate self-responding dimensions (CS; ie, self-kindness, mindfulness, and common humanity), and three uncompassionate self-responding dimensions (UCS; ie, self-judgment, isolation, and overidentification). The negative dimensions were reverse coded, given self-compassion involves concomitantly engaging in CS and disengaging in UCS. The mean of six subscales was averaged and created an index of selfcompassion. An example item for SCS-SF is "When I'm going through a very hard time, I give myself the caring and tenderness I need." SCS-SF was validated in Chinese college students, with good psychometric properties. ${ }^{35}$ In the represent study, SCS-SF shows high internal consistency reliability (Cronbach's $\alpha=0.86$ ). The confirmatory 
factor analysis (CFA) indicated acceptable model fits, $\chi^{2}=$ 634.18, $d f=39, p<0.001, \mathrm{CFI}=0.95$, TLI $=0.91$, RMSEA $=0.09$, and SRMR $=0.06$, suggesting adequate structural validity. According to a recommended cutoff point (3.5) for the scale, ${ }^{36}$ participants were classified into low-level SC (LSC) and high-level SC (HSC), respectively.

\section{Depression Symptoms}

The severity of depression symptoms was measured by the Chinese version of the 9-item Patient Health Questionnaire (PHQ-9). ${ }^{37}$ Each item is reported on a 4-point Likert scale $(0=$ "Not at all" to $3=$ "Nearly every day"). An example item is "Feeling down, depressed, or hopeless." Higher scores indicated more severe depression symptoms. Existed study showed that individuals with a total score of 10 or above were indicated as having probable major depression, ${ }^{38}$ and thus participants were classified into two categories: with depression symptoms (PHQ-9 score $\geq 10$ ) and without depression symptoms (PHQ-9 score $<10$ ). PHQ-9 is validated in Chinese college students with good psychometric properties. ${ }^{39}$ The Cronbach's $\alpha$ coefficient of PHQ-9 in the study is 0.90 . The CFA showed good model fits, $\chi^{2}=407.57, d f=25, p<0.001, \mathrm{CFI}=0.96$, TLI $=$ 0.94 , RMSEA $=0.91$, and SRMR $=0.03$, indicating good structural validity.

\section{Statistical Analyses}

We analyzed data in 4 steps. First, descriptive statistical analyses were conducted. Given the data was collected based on self-reported measures, the Harman singlefactor method was performed to test common method biases. ${ }^{40}$ Second, logistic regression models were used to examine the separate association of LPA and LSC with depression symptoms. With HPA and HSC as referent, odds of depression symptoms for LPA and LSC were presented by odds ratios (ORs) and $95 \%$ confidence intervals (CIs). We mutually adjusted exposures (LPA and LSC) for each other (ie, when LPA was modeled as the main exposure, the analysis was adjusted for LSC, and when LSC was modeled as the main exposure, the analysis was adjusted for LPA).

Third, we examined if the joint association of LPA and LSC with depression symptoms was larger than the sum of the separate associations of LPA and LSC with depression symptoms. Study samples were divided into $4(2 \times 2)$ groups (ie, Group I: HPA + HSC; Group II: LPA + HSC; Group III: HPA + LSC; Group IV: LPA + LSC). If LPA was present, then $i=1$; otherwise $i=0$. If LSC was present, then $j=1$; otherwise $j=0$. Next, a logistic regression model was conducted to computed the estimates and $95 \% \mathrm{CI}$ of $\mathrm{OR}_{\mathrm{ij}}$ to represent the odds of depression symptoms in each category (ie, $\mathrm{OR}_{00}$ [reference category], $\mathrm{OR}_{10}, \mathrm{OR}_{01}, \mathrm{OR}_{11}$ ).

Fourth, the potential additive interaction association between LPA and LSC with depression symptoms was explored. Measures regarding relative excess risk due to interaction (RERI), attributable proportion (AP), and synergy index (S) were calculated. ${ }^{41,42}$ A growing body of studies showed that the additive scale instead of the multiplicative scale is more important in public health assessment, given it allows people to distinguish whether the effects are different in specific subgroups. ${ }^{43,44}$ Therefore, for disease prevention and health promotion among the vulnerable populations, the additive interaction is more suitable than the multiplicative scale. ${ }^{41}$ Therefore, we assessed the presence of interaction between LPA and LSC on the additive scale using the algorithm developed by Anderson et al. ${ }^{42}$ If the $95 \%$ CIs of RERI and AP did not contain 0 and the $95 \% \mathrm{CI}$ of $\mathrm{S}$ did not contain 1 , it can be considered that there is an additive interaction between LPA and LSC. The 95\% CIs were calculated based on 20,000 bootstrap samples.

In all models, the following variables were controlled: age, gender, BMI, number of siblings, residence, family structure, and number of friends. Statistical analyses were performed using SPSS software (IBM SPSS 23.0, SPSS Inc). Significance was set as $p<0.05$.

\section{Results}

Among the 1846 participants (mean age: $20.67 \pm 1.61$, $64.0 \%$ females), $51.5 \%$ and $73.0 \%$ were classified to LPA and LSC, respectively. In total, $23.6 \%$ of participants were screened positive for depression symptoms in this study. More details of participants are listed in Table 1. The results of Harman single-factor analysis showed that the variance interpretation rate of the first common factor was $25.92 \%$, less than the critical standard of $40 \%$, indicating no serious common method bias in this study. ${ }^{40}$

Table 2 presents the independent association of LPA and LSC with depression symptoms. Compared with those with HPA, participants with LPA were more likely to report depression symptoms $(\mathrm{OR}=1.47,95 \% \mathrm{CI}=$ 1.16-1.86) after adjusting for socio-demographic variables and SC (confirmation of Hypothesis 1a). Likewise, compared with those with HSC, LSC was associated with 
Table I The Characteristics of Participants

\begin{tabular}{|c|c|c|c|}
\hline & $\mathbf{N}(\%) / M \pm S D$ & & $N(\%) / M \pm S D$ \\
\hline Gender & & Residence & \\
\hline Male & $665(36.0)$ & Urban & $1278(69.2)$ \\
\hline Female & II8I (64.0) & Rural & $568(30.8)$ \\
\hline Age (years) & $20.67 \pm 1.61$ & Physical activity & \\
\hline Body mass index $\left(\mathrm{kg} / \mathrm{m}^{2}\right)$ & $20.26 \pm 2.88$ & LPA & $950(51.5)$ \\
\hline Family structure & & HPA & $896(48.5)$ \\
\hline Full & $1664(90.2)$ & Self-compassion & \\
\hline Divorced & $117(6.3)$ & LSC & I $347(73.0)$ \\
\hline Other & $65(3.5)$ & HSC & $499(27.0)$ \\
\hline Number of siblings & & Groups & \\
\hline None & $639(34.6)$ & $\mathrm{HPA}+\mathrm{HSC}$ & $288(15.6)$ \\
\hline One or more & $1207(65.4)$ & LPA + HSC & $211(11.4)$ \\
\hline Number of friends & & HPA + LSC & $608(32.9)$ \\
\hline None & $29(1.6)$ & LPA + LSC & $739(40.0)$ \\
\hline One to two & $608(32.9)$ & Depression symptoms & \\
\hline Three to five & $964(52.2)$ & With symptoms & $425(23.6)$ \\
\hline Six or more & $245(13.3)$ & Without symptoms & 14||$(76.4)$ \\
\hline
\end{tabular}

Abbreviations: HPA/LPA, high- or low-level physical activity; HSC/LSC, high- or low-level self-compassion.

Table 2 Independent Association of Physical Activity and Self-Compassion with Depression Symptoms

\begin{tabular}{|c|c|c|c|}
\hline & & OR & $95 \% \mathrm{Cl}$ \\
\hline \multirow[t]{2}{*}{ Gender } & Male & (ref) & \\
\hline & Female & $1.44 * *$ & $1.11-1.87$ \\
\hline Age & & 1.00 & $0.93-1.07$ \\
\hline Body mass index & & 0.98 & $0.94-1.02$ \\
\hline \multirow[t]{3}{*}{ Family structure } & Full & (ref) & \\
\hline & Divorced & 0.85 & $0.51-1.40$ \\
\hline & Other & 1.38 & $0.79-2.42$ \\
\hline \multirow[t]{2}{*}{ Number of siblings } & None & (ref) & \\
\hline & One or more & 1.12 & $0.87-1.44$ \\
\hline \multirow[t]{4}{*}{ Number of friends } & None & (ref) & \\
\hline & One to two & $0.31 * *$ & $0.14-0.68$ \\
\hline & Three to five & $0.22^{* * *}$ & $0.10-0.47$ \\
\hline & Six or more & $0.17^{* * *}$ & $0.07-0.40$ \\
\hline \multirow[t]{2}{*}{ Residence } & Rural & (ref) & \\
\hline & Urban & 0.91 & $0.7 I-1.18$ \\
\hline \multirow[t]{2}{*}{ Physical activity } & LPA & (ref) & \\
\hline & HPA & $1.47 * *$ & $1.16-1.86$ \\
\hline \multirow[t]{2}{*}{ Self-compassion } & LSC & (ref) & \\
\hline & HSC & $5.74 * * *$ & $3.89-8.45$ \\
\hline
\end{tabular}

Notes: $* * p<0.01$, ***p $<0.001$.

Abbreviations: HPA/LPA, high- or low-level physical activity; HSC/LSC, high- or low-level self-compassion. 
Table 3 Logistic Regression Results of Joint Association Between Physical Activity and Self-Compassion with Depression Symptoms

\begin{tabular}{|l|l|l|l|}
\hline LPA & LSC & B & OR (95\% CI) \\
\hline 0 & 0 & - & 1 \\
1 & 0 & 0.63 & $1.88(0.90-3.96)$ \\
0 & 1 & 1.92 & $6.82(3.77-12.33)$ \\
1 & 1 & 2.26 & $9.62(5.38-17.22)$ \\
\hline
\end{tabular}

Notes: $0=$ No exposure; $\mathrm{I}$ = Exposure. Categorical variables are dummy coded before entering the logistic regression model. Results adjusted for gender, age, body mass index, family structure, number of siblings, number of friends, and residence. Abbreviations: LPA, low-level physical activity; LSC, low-level self-compassion.

higher odds for depression symptoms $(\mathrm{OR}=5.74,95 \% \mathrm{CI}$ $=3.89-8.45)$ after adjustment for socio-demographic variables and PA (confirmed Hypothesis 1b).

Table 3 shows the joint association of LPA and LSC with depression symptoms after adjusting for sociodemographic variables. With Group I (HPA + HSC) as the referent, the OR of Group IV (LPA + LSC) reached 9.62 (95\% CI $=5.28-17.22)$, which indicated that, for individuals with concurrent LPA and LSC, the risk for depression symptoms was nearly ten times as those with both HPA and HSC (confirmation of Hypothesis 2).

Table 4 displays the presence of the additive association of LPA and LSC with depression symptoms. Specifically, both RERI and S were significant, indicating the LPA and LSC had a synergic effect on the occurrence of depression symptoms in this sample (confirmation of Hypothesis 3). AP was 0.25 (95\% CI: 0.05-0.44), suggesting that $25 \%$ of depression symptoms may be due to the interaction of LPA and LSC.

\section{Discussion}

\section{Main Findings}

This study examined the independent, joint, and additive interaction associations between LPA and LSC with depression symptoms among Chinese college students.

Table 4 Index of Additive Interaction Between Physical Activity and Self-Compassion on Depression Symptoms

\begin{tabular}{|l|l|l|}
\hline Index & Estimate & $\mathbf{9 5 \%} \mathbf{~ C l}$ \\
\hline RERI & 2.47 & $0.19-4.75$ \\
AP & 0.25 & $0.05-0.44$ \\
S & 1.38 & $1.02-1.87$ \\
\hline
\end{tabular}

Note: Adjusted for gender, age, body mass index, family structure, number of siblings, number of friends, and residence.

Abbreviations: RERI, relative excess risk due to interaction; AP, proportion attributable to the interaction; S, synergy index.
Our findings showed that LPA and LSC were independently associated with depression symptoms. Furthermore, those who engaged in both LPA and LSC had a higher risk of depression symptoms than those who had none or one of these two behaviors. We also found that LPA and LSC synergistically increased the odds of depression symptoms (additive interaction), suggesting that the joint association was more significant than the sum of the separate association. Interpretation of these results is as follows.

\section{Interpretations of Findings}

The study found that LPA was associated with an increased risk of depression symptoms, which supported previous studies on young adults in the context of China and other countries. ${ }^{45,46}$ Similar findings across the world suggest that PA is a globally applicable relevant factor in depression symptoms. The antidepressant effects of PA can be explained from two aspects: biological and psychological mechanisms. ${ }^{47}$ Regarding the biological mechanism, it is suggested that PA elicits a wide range of structural and functional changes in the brain, which suggests that PA may produce its effect through multiple pathways, such as balancing neurotransmitters related to mood-regulation or stress response (eg, cortisol). ${ }^{48,49}$ PA was also associated with the decrease in basal proinflammatory markers and the increase in antiinflammatory markers. ${ }^{50}$ Thus, PA can moderate the relationship between inflammation and depression symptoms. ${ }^{51}$ In terms of the psychological mechanism, PA improves self-esteem and self-efficacy, for example, by improving physical self-perceptions and transferable sense of coping with challenges. ${ }^{52,53}$ PA also makes it more accessible for individuals to get more social support by more interaction and emotional disclosure during exercises with companions. ${ }^{54,55}$ Besides, physically active individuals are prone to have other healthy behaviors in their daily life, such as recommended eating and sleep habits, which are also associated with better mental health. ${ }^{56-59}$ Therefore, compared to the participants with HPA who could obtain the benefits of PA above, it is not surprising to find those with LPA had a greater risk for depression symptoms in this study.

We also found that LSC significantly increased the risk of depression symptoms, which supported previous studies. ${ }^{16,60}$ Studies across age and populations showed that SC could strongly affect overall well-being. ${ }^{61,62}$ The potential effect of SC may also be explained from physiological and psychological perspectives. SC is associated 
with the mammalian caring systems. ${ }^{63}$ The activation of this system may generate oxytocin, which can downregulate one's stress response and alleviate mood disorders (eg, depression). ${ }^{64}$ Besides, SC indicated a competence to treat oneself with kindness when suffering pain. ${ }^{65}$ Conversely, individuals with LSC may manifest a high level of self-criticism. ${ }^{66}$ Many studies showed that excessive self-criticism might lead to depression symptoms. ${ }^{67}$ One possible reason is that self-criticism, similar to external criticism, may diminish one's self-esteem and generate a sense of failure, leading to depression symptoms. ${ }^{68}$ In addition, individuals with LSC tend to ruminate on the sufferings and setbacks they experienced, which also explain the development of depression symptoms. ${ }^{69}$

Notably, a substantial increase in the odds of depression symptoms was observed in combination with both LPA and LSC in this study. Our findings partially support previous research demonstrating that the increase in the number of unhealthy behaviors increased the odds for depression symptoms. ${ }^{70-72}$ Our findings are also in line with the cumulative risk model, suggesting that cumulative risk predicts cumulative outcome. ${ }^{19}$ As expected, both LPA and LSC were risk factors for depression symptoms, and thus their co-occurrence could significantly increase the risk of the symptoms.

Moreover, the combination of LPA and LSC presented a synergy effect on the association with depression symptoms after adjusting for covariates in this study. In other words, the coexistence of LPA and LSC may make an additive risk on depression symptoms. Although underlying mechanisms of this finding can hardly be fully classified based on our data, some empirical evidence may be helpful in explaining the additive interaction. Research suggests that individuals with LPA are more prone to have rumination and self-coldness, which may negatively impact their capacity of $\mathrm{SC}^{73,74}$ and trigger psychological problems such as depression. ${ }^{75}$ Individuals with LSC may have symptoms of self-isolation that may affect their sense of relatedness, and LSC may also reduce individuals' autonomous health behaviors both psychologically and physiologically. These, subsequently, may lead to emotional dysregulation ${ }^{76}$ and learned helplessness, ${ }^{77}$ which may further undermine selfefficacy and increase the likelihood of experiencing depression symptoms. Taken together, these findings may partly explain why additive interaction between LPA and LSC act synergistically to increase the odds of depression symptoms. Nevertheless, literature concerning the additive association between LPA and LSC on depression symptoms is rare, more research is called in the area to clarify the mechanisms.

\section{Limitations and Strengths}

This study has several limitations. First, the cross-sectional design did not allow us to infer causality. Second, this study collected data from university students, mainly from Guangdong province. The results may not be generalizable to other populations. Third, the assessments of PA, $\mathrm{SC}$, and depression symptoms in this study were selfreported, leading to potential recall bias. Fourth, after adjusting for socio-demographic factors, even though the indexes of additive interaction of physical activity and self-compassion were still significant, the lower confidence intervals of AP and $\mathrm{S}$ are close to the statistical rejection area. This suggested that the significance may be marginal. Nevertheless, we still find $25 \%$ of depression symptoms attributed to the interaction of LPA and LSC. Future studies are needed to validate or refute our findings. Finally, it is worth to be noted that PA is the superordinate concept of exercise. Exercise refers to several kinds of PA that are planned, structured for purposes of fitness. ${ }^{78}$ More purposed and moderate-to-high intensity exercise, relative to incidental PA, may pose a distinct influence on depression symptoms and differently interact with SC, whereas it is not precisely measured in this study. But generally, college students accompanied with LPA and LSC were prone to have more depression symptoms. Future studies can further improve the study design with more precision.

Despite these limitations, the present study investigates the relationship between $\mathrm{PA}$ and $\mathrm{SC}$ with depression among Chinese college students based on large sample size. One of the significant strengths of this study was its novelty in examining the additive association between PA and SC. The current study results highlight the potential interplay between PA and SC in the prevention or intervention of depression symptoms among college students. The finding provides some practical implications. Specifically, concurrently increasing participation in PA and SC may provide more effective prevention and intervention for depression symptoms. A growing body of evidence has indicated that adding psychological components (eg, mindfulness) to exercise-based interventions could contribute to better effects. ${ }^{79-81}$ For example, Kratz et $\mathrm{al}^{82}$ found that the exercise-based intervention combined with psychological treatment may be more effective for individuals with depression symptoms than simple exercise intervention. Likewise, $\mathrm{Li}$ et $\mathrm{al}^{83}$ found that mindful 
exercise was more beneficial to reduce psychiatric symptoms than pure aerobic exercise. According to recent metaanalyses, ${ }^{84,85}$ body-mind exercises were of great benefit to treating depression symptoms. Therefore, clinical measures integrating PA and SC can also be designed and taken to help prevent or reduce depression symptoms in young adults. For instance, when we design the interventions for depressive symptoms, we could add some SC components to PA-based interventions or some PA components to SC-based interventions. The effect of these interventions may be better than the interventions involving only PA or SC.

\section{Conclusions}

LPA and LSC could independently increase the odds of depression symptoms among college students and act synergistically to increase the odds of depression symptoms with an additive interaction. To find a better way to resist the risk of depression symptoms, future research and clinical intervention could integrate PA and SC.

\section{Abbreviations}

PA, physical activity; SC, self-compassion; LPA, low-level physical activity; LSC, low-level self-compassion; RERI, relative excess risk due to interaction; $\mathrm{AP}$, attributable proportion; S, synergy index; OR, odds ratio.

\section{Data Sharing Statement}

Available upon request to the first author.

\section{Ethics Approval and Informed Consent}

The Human Research Ethics Committee of Shenzhen university granted ethics approval (Approval Number: 2020005), and that it was conducted in accordance with the Declaration of Helsinki.

\section{Consent for Publication}

All authors consent for publication.

\section{Acknowledgment}

We are very grateful to our colleagues for their diligent work, the participants who took the time to participate in this study, and their teachers and parents to promote their participation.

\section{Funding}

$\mathrm{XC}$ was supported by the Guangdong Basic and Applied Basic Research Foundation (Grant No.2021A1515011330). YZ was funded by the Shenzhen Science and Technology Research and Development Funds of the Shenzhen Science and Technology Innovation Committee (Grant No.20200814102701001).

\section{Disclosure}

The authors report no conflicts of interest in this work.

\section{References}

1. Jiang CX, Li ZZ, Chen P, Chen LZ. Prevalence of depression among college-goers in Mainland China: a methodical evaluation and meta-analysis. Medicine. 2015;94(50):e2071. doi:10.1097/ MD.0000000000002071

2. World Health Organization. Depression. Vol 10/ 16/2020; 2020.

3. Rao AL, Hong ES. Understanding depression and suicide in college athletes: emerging concepts and future directions. $\mathrm{Br} J$ Sport Med. 2015;50(3):136. doi:10.1136/bjsports-2015-095658

4. Firth J, Ward PB, Stubbs B. Editorial: lifestyle psychiatry. Front Psychiatry. 2019;10:597. doi:10.3389/fpsyt.2019.00597

5. Noordsy DL. Lifestyle Psychiatry. American Psychiatric Pub; 2019.

6. Schuch FB, Vancampfort D, Firth J, et al. Physical activity and incident Depression: a meta-analysis of prospective cohort studies. Am J Psychiatry. 2018;175(7):631-648. doi:10.1176/appi. ajp.2018.17111194

7. Taliaferro LA, Rienzo BA, Pigg RM, Miller MD, Dodd VJ. Associations between physical activity and reduced rates of hopelessness, depression, and suicidal behavior among college students. $J \mathrm{Am}$ Coll Health. 2009;57(4):427-436. doi:10.3200/JACH.57.4.427-436

8. Sfendla A, Hadrya F. Factors associated with psychological distress and physical activity during the COVID-19 pandemic. Health Secur. 2020;18(6):444-453. doi:10.1089/hs.2020.0062

9. Fu W, Wang C, Zou L, et al. Psychological health, sleep quality, and coping styles to stress facing the COVID-19 in Wuhan, China. Transl Psychiatry. 2020;10(1):1-9. doi:10.1038/s41398-020-00913-3.

10. Mikkelsen K, Stojanovska L, Polenakovic M, Bosevski M, Apostolopoulos V. Exercise and mental health. Maturitas. 2017;106:48-56. doi:10.1016/j.maturitas.2017.09.003

11. Merikangas KR, Swendsen J, Hickie IB, et al. Real-time mobile monitoring of the dynamic associations among motor activity, energy, mood, and sleep in adults with bipolar disorder. JAMA Psychiatry. 2019;76(2):190-198. doi:10.1001/jamapsychiatry.2018.3546

12. Hagger MS, Chatzisarantis NLD. Integrating the theory of planned behaviour and self-determination theory in health behaviour: a meta-analysis. $\mathrm{Br} \quad J$ Health Psychol. 2009;14(2):275-302. doi:10.1348/135910708X373959

13. Codella R, Puci MV, Vandoni M, et al. School self-efficacy is affected by gender and motor skills: findings from an Italian study. Peer J. 2020;8:e8949. doi:10.7717/peerj.8949

14. Neff KD, Dahm KA. Self-compassion: what it is, what it does, and how it relates to mindfulness. In: Handbook of Mindfulness and SelfRegulation. Springer; 2015:121-137.

15. Ferrari M, Hunt C, Harrysunker A, Abbott MJ, Beath AP, Einstein DA. Self-compassion interventions and psychosocial outcomes: a meta-analysis of RCTs. Mindfulness. 2019;10 (8):1455-1473. doi:10.1007/s12671-019-01134-6

16. MacBeth A, Gumley A. Exploring compassion: a meta-analysis of the association between self-compassion and psychopathology. Clin Psychol Rev. 2012;32(6):545-552. doi:10.1016/j.cpr.2012.06.003 
17. Kaurin A, Schönfelder S, Wessa M. Self-compassion buffers the link between self-criticism and depression in trauma-exposed firefighters. J Couns Psychol. 2018;65(4):453. doi:10.1037/cou0000275

18. Aruta JJBR, Antazo B, Briones-Diato A, Crisostomo K, Canlas NF, Peñaranda $\mathrm{G}$. When does self-criticism lead to depression in collectivistic context. Int $J$ Adv Couns. 2021;43(1):76-87. doi:10.1007/ s10447-020-09418-6

19. Atkinson L, Beitchman J, Gonzalez A, et al. Cumulative risk, cumulative outcome: a 20-year longitudinal study. PLoS One. 2015;10(6): e0127650. doi:10.1371/journal.pone.0127650

20. Doan SN, Fuller-Rowell TE, Evans GW. Cumulative risk and adolescent's internalizing and externalizing problems: the mediating roles of maternal responsiveness and self-regulation. Dev Psychol. 2012;48(6):1529-1539. doi:10.1037/a0027815

21. Sirois FM, Kitner R, Hirsch JK. Self-compassion, affect, and health-promoting behaviors. Health Psychol. 2015;34(6):661. doi:10.1037/hea0000158

22. Cox AE, Ullrich-French S, Tylka TL, McMahon AK. The roles of self-compassion, body surveillance, and body appreciation in predicting intrinsic motivation for physical activity: cross-sectional associations, and prospective changes within a yoga context. Body Image. 2019;29:110-117. doi:10.1016/j.bodyim.2019.03.002

23. Magnus CMR, Kowalski KC, McHugh TF. The role of selfcompassion in women's self-determined motives to exercise and exercise-related outcomes. Self Identity. 2010;9(4):363-382. doi:10.1080/15298860903135073

24. Warburton DER. Health benefits of physical activity: the evidence. Can Med Assoc J. 2006;174(6):801-809. doi:10.1503/cmaj.051351

25. Conn VS. Depressive symptom outcomes of physical activity interventions: meta-analysis findings. Ann Behav Med. 2010;39 (2):128-138. doi:10.1007/s12160-010-9172-x

26. Mothes H, Klaperski S, Seelig H, Schmidt S, Fuchs R. Regular aerobic exercise increases dispositional mindfulness in men: a randomized controlled trial. Ment Health Phys Act. 2014;7 (2):111-119. doi:10.1016/j.mhpa.2014.02.003

27. Wong MYC, Chung P, Leung K. The relationship between physical activity and self-compassion: a systematic review and meta-analysis. Mindfulness. 2021;12(3):547-563. doi:10.1007/s12671-020-01513-4

28. Chi X, Becker B, Yu Q, et al. Prevalence and psychosocial correlates of mental Health outcomes among Chinese college students during the Coronavirus disease (COVID-19) pandemic. Front Psychiatry. 2020;11:803.

29. Schuch FB, Bulzing RA, Meyer J, et al. Associations of moderate to vigorous physical activity and sedentary behavior with depressive and anxiety symptoms in self-isolating people during the COVID-19 pandemic: a cross-sectional survey in Brazil. Psychiatry Res Neuroimaging. 2020;292:113339. doi:10.1016/j. psychres.2020.113339

30. Faul F, Erdfelder E, Buchner A, Lang A. Statistical power analyses using $G^{*}$ Power 3.1: tests for correlation and regression analyses. Behav Res Methods. 2009;41(4):1149-1160. doi:10.3758/ BRM.41.4.1149

31. Faul F, Erdfelder E, Lang AG, Buchner A. G*Power 3: a flexible statistical power analysis program for the social, behavioral, and biomedical sciences. Behav Res Methods. 2007;39(2):175-191. doi:10.3758/BF03193146

32. Craig CL, Marshall AL, Sjöström M, et al. International physical activity questionnaire: 12-country reliability and validity. Med Sci Sport Exerc. 2003;35(8):1381-1395. doi:10.1249/01. MSS.0000078924.61453.FB

33. Macfarlane DJ, Lee CCY, Ho EYK, Chan KL, Chan DTS. Reliability and validity of the Chinese version of IPAQ (short, last 7 days). $J \mathrm{Sci}$ Med Sport. 2007;10(1):45-51. doi:10.1016/j.jsams.2006.05.003

34. Raes F, Pommier E, Neff KD, Van Gucht D. Construction and factorial validation of a short form of the self-compassion scale. Clin Psychol Psychother. 2011;18(3):250-255. doi:10.1002/cpp.702
35. Huang L, Qu D, Liang K, Ren Y, Chi X. Longitudinal analysis of the self-compassion scale-short form in Chinese college students. Chin $J$ Clin Psychol. 2021. Under Review.

36. Neff KD. The development and validation of a scale to measure self-compassion. Self Identity. 2003;2(3):223-250. doi:10.1080/ 15298860309027

37. Li Y, Wang X, Zhang J, Du S, Zeng L. Psychological survey of COVID-19 pandemic among the general public. Infect Int. 2020;9(2):308-310.

38. Levis B, Benedetti A, Thombs BD. Accuracy of Patient Health Questionnaire-9 (PHQ-9) for screening to detect major depression: individual participant data meta-analysis. BMJ. 2019;(365):11476. doi:10.1136/bmj.11476

39. Du N, Yu K, Ye Y, Chen S. Validity study of Patient Health Questionnaire-9 items for Internet screening in depression among Chinese university students. Asia Pac Psychiatry. 2017;9(3):e12266. doi:10.1111/appy.12266

40. Podsakoff PM, MacKenzie SB, Lee JY, Podsakoff NP. Common method biases in behavioral research: a critical review of the literature and recommended remedies. $J$ Appl Psychol. 2003;88 (5):879-903. doi:10.1037/0021-9010.88.5.879

41. Knol MJ, VanderWeele TJ. Recommendations for presenting analyses of effect modification and interaction. Int $J$ Epidemiol. 2012;41(2):514-520. doi:10.1093/ije/dyr218

42. Andersson T, Alfredsson L, Källberg H, Zdravkovic S, Ahlbom A. Calculating measures of biological interaction. Eur J Epidemiol. 2005;20(7):575-579. doi:10.1007/s10654-005-7835-x

43. Knol MJ, VanderWeele TJ, Groenwold RHH, Klungel OH, Rovers MM, Grobbee DE. Estimating measures of interaction on an additive scale for preventive exposures. Eur J Epidemiol. 2011;26 (6):433-438. doi:10.1007/s10654-011-9554-9

44. Rothman KJ, Greenland S, Walker AM. Concepts of interaction. Am $J$ Epidemiol. 1980;112(4):467-470. doi:10.1093/oxfordjournals.aje. a113015

45. Feng Q, Zhang Q, Du Y, Ye Y, He Q, Stewart R. Associations of physical activity, screen time with depression, anxiety and sleep quality among Chinese college freshmen. PLoS One. 2014;9(6): e100914. doi:10.1371/journal.pone.0100914

46. De Mello MT, Lemos VDA, Antunes HKM, Bittencourt L, SantosSilva R, Tufik S. Relationship between physical activity and depression and anxiety symptoms: a population study. J Affect Disord. 2013;149(1-3):241-246. doi:10.1016/j.jad.2013.01.035

47. Kandola A, Ashdown-Franks G, Hendrikse J, Sabiston CM, Stubbs B. Physical activity and depression: towards understanding the antidepressant mechanisms of physical activity. Neurosci Biobehav Rev. 2019;107:525-539.

48. Budde H, Machado S, Ribeiro P, Wegner M. The cortisol response to exercise in young adults. Front Behav Neurosci. 2015;9:13.

49. Heijnen S, Hommel B, Kibele A, Colzato LS. Neuromodulation of aerobic exercise-A review. Front Psychol. 2016;6:1890.

50. Codella R, Chirico A, Lucidi F, Ferrulli A, La Torre A, Luzi L. The immune-modulatory effects of exercise should be favorably harnessed against COVID-19. J Endocrinol Invest. 2021;44 (5):1119-1122. doi:10.1007/s40618-020-01403-5

51. Werneck AO, Christofaro DGD, Ritti-Dias RM, et al. Does physical activity influence the association between depressive symptoms and low-grade inflammation in adults? A study of 8048 adults. Physiol Behav. 2020;223:112967. doi:10.1016/j.physbeh.2020.112967

52. Moore JB, Mitchell NG, Bibeau WS, Bartholomew JB. Effects of a 12-week resistance exercise program on physical self-perceptions in college students. Res $Q$ Exerc Sport. 2011;82(2):291-301. doi:10.1080/02701367.2011.10599757

53. Oddie S, Fredeen D, Williamson B, DeClerck D, Doe S, Moslenko K. Can physical activity improve depression, coping \& motivation to exercise in children and youth experiencing challenges to mental wellness? Psychology. 2014;05(19):2147-2158. doi:10.4236/ psych.2014.519217 
54. Hernáez AM, Maicas NC, DiGiacomo SM, Ariste S. Social support and gender differences in coping with depression among emerging adults: a mixed - methods study. Child Adolesc Psychiatry Ment Health. 2016;10(1):1.

55. Kouvonen A, De Vogli R, Stafford M, et al. Social support and the likelihood of maintaining and improving levels of physical activity: the Whitehall II Study. Eur J Public Health. 2012;22(4):514-518. doi:10.1093/eurpub/ckr091

56. Weng T, Hao J, Qian Q, et al. Is there any relationship between dietary patterns and depression and anxiety in Chinese adolescents? Public Health Nutr. 2012;15(4):673-682. doi:10.1017/ S1368980011003077

57. Ball K, Jeffery RW, Abbott G, McNaughton SA, Crawford D. Is healthy behavior contagious: associations of social norms with physical activity and healthy eating. Int J Behav Nutr Phys Act. 2010;7 (1):86. doi:10.1186/1479-5868-7-86

58. Lang C, Kalak N, Brand S, Holsboer-Trachsler E, Pühse U, Gerber M. The relationship between physical activity and sleep from mid adolescence to early adulthood. A systematic review of methodological approaches and meta-analysis. Sleep Med Rev. 2016;28:32-45. doi:10.1016/j.smrv.2015.07.004

59. Orchard F, Gregory AM, Gradisar M, Reynolds S. Self-reported sleep patterns and quality amongst adolescents: cross-sectional and prospective associations with anxiety and depression. J Child Psychol Psychol. 2020;61(10):1126-1137. doi:10.1111/jcpp.13288

60. Marsh IC, Chan SWY, MacBeth A. Self-compassion and psychological distress in adolescents - a meta-analysis. Mindfulness. 2018;9 (4):1011-1027. doi:10.1007/s12671-017-0850-7

61. Phillips WJ, Hine DW. Self-compassion, physical health, and health behaviour: a meta-analysis. Health Psychol Rev. 2019;15(1):1-27.

62. Zessin U, Dickhäuser O, Garbade S. The relationship between self-compassion and well-being: a meta-analysis. Appl Psychol Health Well Being. 2015;7(3):340-364.

63. Braehler C, Neff K. Self-compassion in PTSD. In: Braehler C, Neff K, editors. Emotion in Posttraumatic Stress Disorder. Waltham: Academic Press; 2020:567-596.

64. McQuaid RJ, McInnis OA, Abizaid A, Anisman H. Making room for oxytocin in understanding depression. Neurosci Biobehav Rev. 2014;45:305-322.

65. Neff KD, Long P, Knox MC, et al. The forest and the trees: examining the association of self-compassion and its positive and negative components with psychological functioning. Self Identity. 2018;17 (6):627-645. doi:10.1080/15298868.2018.1436587

66. Bluth K, Neff KD. New frontiers in understanding the benefits of self-compassion. Self Identity. 2018;17(6):605-608. doi:10.1080/ 15298868.2018.1508494

67. Mcintyre R, Smith P, Rimes KA. The role of self-criticism in common mental health difficulties in students: a systematic review of prospective studies. Ment Health Prev. 2018;10:13-27.

68. Chang C, Yuan R, Chen J. Social support and depression among Chinese adolescents: the mediating roles of self-esteem and self-efficacy. Child Youth Serv Rev. 2018;88:128-134. doi:10.1016/j. childyouth.2018.03.001

69. Svendsen JL, Kvernenes KV, Wiker AS, Dundas I. Mechanisms of mindfulness: rumination and self-compassion. Nord Psychol. 2017;69 (2):71-82. doi:10.1080/19012276.2016.1171730

70. Werneck AO, Silva DR, Malta DC, et al. Changes in the clustering of unhealthy movement behaviors during the COVID-19 quarantine and the association with mental health indicators among Brazilian adults. Transl Behav Med. 2021;11(2):323-331. doi:10.1093/tbm/ibaa095
71. Lu C, Chi X, Liang K, et al. Moving more and sitting less as healthy lifestyle behaviors are protective factors for insomnia, depression, and anxiety among adolescents during the COVID-19 pandemic. Psychol Res Behav Manag. 2020;13:1223-1233. doi:10.2147/ PRBM.S284103

72. Cao R, Gao T, Hu Y, et al. Clustering of lifestyle factors and the relationship with depressive symptoms among adolescents in Northeastern China. J Affect Disord. 2020;274:704-710. doi:10.1016/j.jad.2020.05.064

73. Babenko O, Mosewich A, Abraham J, Lai H. Contributions of psychological needs, self-compassion, leisure-time exercise, and achievement goals to academic engagement and exhaustion in Canadian medical students. J Educ Eval Health Prof. 2018;15:2. doi:10.3352/jeehp.2018.15.2

74. Décamps G, Boujut E, Brisset C. French college students' sports practice and its relations with stress, coping strategies and academic success. Front Psychol. 2012;3:104. doi:10.3389/fpsyg.2012.00104

75. Vandenkerckhove B, Soenens B, Flamant N, Luyten P, Campbell R, Vansteenkiste M. Daily ups and downs in adolescents' depressive symptoms: the role of daily self-criticism, dependency and basic psychological needs. J Adolesc. 2021;91:97-109. doi:10.1016/j. adolescence.2021.07.005

76. Guo T, Huang L, Hall DL, et al. The relationship between childhood adversities and complex posttraumatic stress symptoms: a multiple mediation model. Eur J Psychotraumatol. 2021;12(1):1936921. doi:10.1080/20008198.2021.1936921

77. Seligman MEP. Helplessness: On Depression, Development, and Death. Henry Holt \& Co; 1975.

78. Caspersen CJ, Powell KE, Christenson GM. Physical activity, exercise, and physical fitness: definitions and distinctions for health-related research. Public Health Rep. 1985;100(2):126-131.

79. Cozzolino M, Vivo DR, Celia G. School-based mind-body interventions: a research review. Hu Arenas. 2021:1-7. doi:10.1007/s42087020-00163-1

80. Gautam S, Tolahunase M, Kumar U, Dada R. Impact of yoga based mind-body intervention on systemic inflammatory markers and co-morbid depression in active Rheumatoid arthritis patients: a randomized controlled trial. Restor Neurol Neurosci. 2019;37 (1):41-59.

81. Cozzolino M, Girelli L, Vivo DR, Limone P, Celia G. A mind-body intervention for stress reduction as an adjunct to an information session on stress management in university students. Brain Behav. 2020;10(6):e1651. doi:10.1002/brb3.1651

82. Kratz AL, Ehde DM, Bombardier CH. Affective mediators of a physical activity intervention for depression in multiple sclerosis. Rehabil Psychol. 2014;59(1):57-67. doi:10.1037/a0035287

83. Li J, Shen J, Wu G, et al. Mindful exercise versus non-mindful exercise for schizophrenia: a systematic review and meta-analysis of randomized controlled trials. Complement Ther Clin. 2018;32:17-24. doi:10.1016/j.ctcp.2018.04.003

84. Zou L, Sasaki JE, Wei G, et al. Effects of mind body exercises (Tai Chi/Yoga) on heart rate variability parameters and perceived stress: a systematic review with meta-analysis of randomized controlled trials. J Clin Med. 2018;7(11):404. doi:10.3390/jcm7110404

85. Zou L, Yeung A, Li C, et al. Effects of meditative movements on major depressive disorder: a systematic review and meta-analysis of randomized controlled trials. J Clin Med. 2018;7(8):195. doi: $10.3390 / \mathrm{jcm} 7080195$ 


\section{Publish your work in this journal}

Risk Management and Healthcare Policy is an international, peerreviewed, open access journal focusing on all aspects of public health, policy, and preventative measures to promote good health and improve morbidity and mortality in the population. The journal welcomes submitted papers covering original research, basic science, clinical \& epidemiological studies, reviews and evaluations, guidelines, expert opinion and commentary, case reports and extended reports. The manuscript management system is completely online and includes a very quick and fair peer-review system, which is all easy to use. Visit http://www.dovepress.com/testimonials.php to read real quotes from published authors. 